\title{
Technical Standards and Guidelines: Molecular Genetic Testing for Ultra-Rare Disorders
}

\author{
Anne Maddalena, $P h D^{1}$, Sherri Bale, $P h D^{1}$, Soma Das, $P h D^{2}$, Wayne Grody, MD, PhD ${ }^{3}$, Sue Richards, $P h D^{4}$, and the
} ACMG Laboratory Quality Assurance Committee

Key Words: clinical genetic testing, sequencing, rare disorders, technical standards and guidelines

\begin{abstract}
Disclaimer: These standards and guidelines are designed primarily as an educational resource for clinical laboratory geneticists to help them provide quality clinical laboratory genetic services. Adherence to these standards and guidelines does not necessarily ensure a successful medical outcome. These standards and guidelines should not be considered inclusive of all proper procedures and tests or exclusive of other procedures and tests that are reasonably directed to obtaining the same results. In determining the propriety of any specific procedure or test, the clinical molecular geneticist should apply his or her own professional judgment to the specific clinical circumstances presented by the individual patient or specimen. It may be prudent, however, to document in the laboratory record the rationale for any significant deviation from these standards and guidelines.
\end{abstract}

One mission of the ACMG Laboratory Quality Assurance (QA) Committee is to develop standards and guidelines for clinical genetics laboratories in an effort to maintain high technical standards for the performance and interpretation of genetic tests. This document was developed by the UltraRare Disorders (URD) Working Group under the auspices of the Molecular Subcommittee of the Laboratory Quality Assurance Committee. It is intended to provide laboratories currently testing for ultra-rare disorders using molecular methods, and those considering expanding their test menus to include one or more such disorders, an overview of the specific issues that arise when performing molecular diagnosis for disorders in which the test is available in only one laboratory or very few laboratories. Specific issues that are addressed include custom mutation analysis and prenatal diagnosis, choice of analytic technique to identify private mutations, concerns about test validation, and interpretation of results. Ultra-rare disorders requiring diagnostic testing using cytogenetic or biochemical analyses are beyond the scope of this guideline and will be addressed in a separate document.

\section{URD 1 INTRODUCTION}

URD 1.1 Under state and federal regulations, only licensed clinical laboratories (i.e., certified under the Clinical Laboratory Improvement Amendments [CLIA]) are legally permitted to perform testing on patients in which the results are released

From ${ }^{1}$ GeneDx, Gaithersburg, MD, ${ }^{2}$ University of Chicago, Chicago, IL, ${ }^{3}$ University of California-Los Angeles, Los Angeles, CA, ${ }^{4}$ Oregon Health Sciences University, Portland, OR American College of Medical Genetics, 9650 Rockville Pike, Bethesda, MD 20814-3998 Go to www.geneticsinmedicine.org for a printable copy of this document.

Approved by the Board of Directors of the American College of Medical Genetics March 16, 2005 DOI: 10.1097/01.GIM.0000182738.95726.ca directly to patients or their physicians and used for medical management. This arrangement works well and is generally adhered to throughout clinical practice, even for esoteric testing areas such as molecular pathology and diagnostic molecular genetics. However, there is one gray area that persists and has recently come under increased scrutiny at the federal level: the transition of testing for ultra-rare disorders from the research setting to the clinical setting. Testing for these disorders, most of which are in the genetic disease area, has evolved this way because: 1) typically only one or two laboratories in the world are studying the causative gene, have recruited affected families for study, and have become expert in the assays; and 2) the diseases are too rare and the test requests too infrequent to generate interest from a financial perspective among high-volume clinical laboratories (hence, the term "ultra-rare" or "orphan" diseases).

URD 1.2 As a relatively new, translational area, lying at the border between research and clinical service, ultra-rare disease molecular genetic testing has few specific national standards for quality assurance, quality control, test accessioning and reporting, or proficiency evaluation. These matters have been discussed by federally mandated agencies, including the $\mathrm{Na}-$ tional Institute of Health (NIH)-Department of Energy (DOE) Task Force on Genetic Testing and the Health and Human Services (HHS) Secretary's Advisory Committee on Genetic Testing. The Task Force in particular devoted an entire portion of its report to concerns over the regulatory status of orphan genetic disease testing, and its final recommendations included somewhat relaxed CLIA requirements for research laboratories providing such services. ${ }^{1}$

URD 1.3 As such tests now begin to make the transition from research laboratories to those clinical laboratories with a willingness to take them on, it has become evident that there is a need for adaptation of existing quality assurance standards in 
molecular diagnostics to ultra-rare disease tests. These standards must be applicable to an almost infinite number of analytes (disease genes and mutations), and should enable the efficient translation of research-based tests to the clinical milieu, the development of informed consent requirements as appropriate, and the construction of versatile tools for quality control, proficiency testing, results interpretation and reporting. The purpose of these guidelines is to address this growing need.

URD 1.4 Evaluation of the Clinical Readiness of Genetic Testing for a Rare Disorder

URD 1.4.1 An important issue in providing molecular diagnostic testing for an ultra-rare disorder is determining when there is enough information to transition a test from the research setting to a clinical diagnostic laboratory. This involves an assessment of the clinical validity and clinical utility of the test. Both clinical validity and clinical utility must be evaluated for each test being considered for translation from a research setting. Unfortunately, assessing these parameters is a challenge for rare disorders because of the paucity of data. However, the extremely low prevalence/incidence of a genetic disorder should not become a roadblock in making genetic testing available to those patients and families for whom testing may provide striking personal, family, and medical benefit. The differences between a diagnostic test for a highly-penetrant disorder (the focus of this document) versus a predictive test for a low-penetrant disorder should be considered.

URD 1.4.2 With that in mind, the following issues should be considered in the determination of a clinical laboratory to offer a molecular test for a rare genetic disorder:

URD 1.4.2.1. Does a review of the literature provide strong evidence of the causative nature of mutations in the implicated gene in the development of the rare genetic disease? (1) Is there significant cosegregation of the gene mutation(s) with the phenotype, and absence of the mutation in clinically-unaffected family members, providing high clinical sensitivity and specificity? (2) Is there evidence from functional studies of the mutated gene product supporting causality with the disease phenotype? and/or (3) Have the findings from the initial reporting laboratory been confirmed by other researchers, or has the data from the initial reporting laboratory included multiple families, groups of families, or patients from more than one population, such that the conclusions can be generalized beyond a very limited set of affected individuals?

URD 1.4.2.2. Do the benefits of a positive or negative test result out-weigh the risks of undergoing the genetic test? Specifically, is there evidence that the genetic test will provide information relevant to diagnosis, prognosis, treatment, medical surveillance, and family planning that will benefit the patient/family having testing?
URD 1.4.3 Data relevant to the continued evaluation of the clinical validity and utility of a genetic test for an ultra-rare disorder will depend on the long-term collection and analysis of additional data gained from clinical testing as well as from further efforts in the research laboratory. Researchers and clinical molecular diagnostic laboratories should be encouraged to share such data as it becomes available.

\section{URD 2 DEFINITIONS}

URD 2.1 Ultra-rare disorder (URD): A disorder with prevalence of fewer than 200,000 individuals in the United States. Some examples include pantothenate kinase-associated neurodegeneration, X-linked ocular albinism, epidermolytic hyperkeratosis, adrenal hypoplasia congenita, ataxia telangiectasia, X-linked myotubular myopathy, lissencephaly, and Menkes disease.

URD 2.2 Mutation: A change from the normal sequence of a gene which underlies a detrimental clinical presentation; a change that is associated with disease.

URD 2.3 Variation: Any change from the normal sequence of a gene. Variations may underlie a disease (e.g., mutation), or may be unrelated to a disease process.

URD 2.4 Confirmation: A repeat analysis on a sample from an individual. This may be done: 1) using a new independent sample; 2) using another aliquot of DNA prepared from the original submitted sample; or 3 ) using another method of analysis from the one used originally to identify the sequence variation.

URD 2.5 Positive control: A DNA sample that harbors a known $\overline{\text { sequence change. }}$

URD 2.6 Negative control: A DNA sample that is known to be normal at the specific sequence position of interest.

URD 2.7 Analytical sensitivity: The proportion of biological samples that have a positive test result or known mutation and that are correctly classified as positive (assumes mutation is tested for).

URD 2.8 Analytical specificity: The proportion of biological samples that have a negative test result or no identified mutation (being tested for) and that are correctly classified as negative.

URD 2.9 Clinical sensitivity: The proportion of individuals who have (or will develop) the phenotype of interest and who have a positive test result.

URD 2.10 Clinical specificity: The proportion of all unaffected individuals identified by the proposed test as being negative. 
URD 2.11 Clinical utility: The value of a test, including risks and benefits, for the purposes of confirmation of diagnosis in a symptomatic patient, presymptomatic analysis, treatment, family planning, and prenatal testing (when relevant).

URD 2.12 Custom analysis: A test for a specific mutation previously identified in a particular family (either in a clinical or research laboratory), not necessarily in a gene for which the laboratory offers broad testing.

\section{URD 3 TECHNOLOGY GUIDELINES}

URD 3.1 The choice in technology is dependent upon the type of mutation and the mutational spectrum of the disorder. Most of the rare disorders are composed of a large mutational spectrum, including a variety of types of mutations in either a single gene or multiple genes. Therefore, a targeted approach to mutational detection for common mutations, such as in cystic fibrosis transmembrane conductance regulator (CFTR) testing, usually is not the case (see "CFTR Mutation Testing" in the Disease/Phenotypic-Specific section of the ACMG Standards and Guidelines for Clinical Genetics Laboratories). For rare disorders, either sequencing the entire coding region and intron/exon boundaries, or a combined approach of scanning for mutations using various platforms and confirming mutations by sequencing, is generally indicated.

URD 3.2 Sequencing

URD 3.2.1 Section G13 of the ACMG Standards and Guidelines for Clinical Genetics Laboratories addresses sequencing.

URD 3.2.2 An excellent adjunct to these guidelines is published by the Clinical and Laboratory Standards Institute (CLSI - formerly NCCLS), entitled MM9-P: Nucleic Acid Sequencing Methods in Diagnostic Laboratory Medicine (December 2004).

URD 3.2.3 For sequencing, it is essential to use sequence analysis software and compare the patient sequence to the wildtype. Inspection of chromatograms for heterozygous positions is insufficient. The software used must support assembly of contigs for analysis, including both forward and reverse directions of fragments sequenced and any overlapping fragments, the cDNA reference sequence, and the genomic reference sequence.

URD 3.2.4 These guidelines address the unique aspects of introducing testing for ultra-rare disorders directly into a CLIAcertified laboratory. Under no circumstances is a CLIA-certified laboratory permitted to utilize a core sequencing facility or other research laboratory to perform this testing.

URD 3.3 Scanning

URD 3.3.1 Sections of the ACMG Standards and Guidelines for Clinical Genetics Laboratories that address scanning tech- nologies include: Denaturing Gradient Gel Electrophoresis (DGGE), G9; Protein Truncation Testing (PTT), G10; Single Strand Conformational Polymorphism (SSCP), G11; Heteroduplex Analysis (HA), G12; and Denaturing High Performance Liquid Chromatography (DHPLC), G18. For scanning technologies, it is important to determine the analytical sensitivity and specificity.

URD 3.4 Limitations of Non-Quantitative Sequencing and Scanning Methods

URD 3.4.1 It is important to understand that these methods will detect point mutations including small deletions and small insertions, nonsense, missense, splicing mutations, single nucleotide polymorphisms (SNPs), and unclassified variants, but they will not detect large gene deletions or duplications or other gross genomic rearrangements.

URD 3.4.2 Therefore, in order to provide comprehensive mutation detection analysis for those genes in which this issue is relevant, it is recommended that the laboratory consider developing a technical platform to identify the presence of such gross alterations. Many such platforms are available, including southern blot, fluorescent in situ hybridization (FISH), multiplex gene dosage analysis, reverse transcription polymerase chain reaction (PCR), long-range PCR, real time quantitative PCR using SYBR ${ }^{\circledR}$ green or fluorescent probe technology, and multiplex ligation probe amplification (MLPA). This is not an inclusive list and continues to evolve. A combination of technologies may be useful as confirmatory testing. Most of these technologies require in-house development of the assay. Thus, it is recommended that only highly experienced laboratories undertake this challenge.

\section{URD 3.5 Linkage Analysis}

URD 3.5.1 In situations in which direct mutation identification may not be possible (e.g., when a critical individual in a family is deceased or otherwise unavailable, or when a multiexon deletion underlies a disorder), it is sometimes possible to use linkage analysis to identify the disease allele segregating in a family, and to use that information for diagnostic and prenatal testing purposes. In such cases, as many informative markers as necessary to identify the disease allele must be typed in all relevant, available family members. Reported results should reflect the uncertainty due to recombination inherent in all linkage-based studies.

URD 3.6 It is important to recognize that the laboratory is responsible for validation of all analytical components of testing (see Section C8 of ACMG Standards and Guidelines for Clinical Genetics Laboratories on Test Validation). 


\section{URD 4 PERSONNEL}

URD 4.1 The standards for laboratory personnel involved in testing for ultra-rare genetic diseases should be essentially equivalent to those in other areas of diagnostic molecular genetic testing, as described in the ACMG Standards and Guidelines for Clinical Genetics Laboratories. Laboratory directors should have a doctoral degree and appropriate training and experience in clinical molecular genetic testing, as evidenced by board certification in clinical molecular genetics, molecular genetic pathology, or equivalent subspecialty expertise. Technical staff should possess sufficient case experience in molecular testing for certification or eligibility by the National Credentialing Agency (NCA) as a molecular biology specialist or equivalent. Since most ultra-rare disease tests are sequencebased, directors and staff should have sufficient experience in the performance and interpretation of DNA sequencing assays for both known and unknown mutations.

\section{URD 5 TEST VALIDATION}

\section{URD 5.1 Clinical Validation of Testing}

URD 5.1.1. The issue of clinical validation in URDs is unique: The accepted guidelines for determining clinical validity for the more common hereditary disorders are not directly applicable due to the paucity of data for any particular rare disorder. It is thus incumbent on each laboratory choosing to add an ultra-rare disorder diagnostic test to its menu to perform a thorough review of all available literature on the gene and mutational spectrum in the disease. Ideally, at least two peer-reviewed publications should provide very strong evidence of the involvement of the particular gene in the development of the disease. In some cases only a single publication with an extensive data set may have been published. Data must be sufficient to determine, with some level of confidence, the sensitivity and specificity of the test to be offered. Particular caution should be taken when considering disorders in which: 1) the phenotype may be due to the interaction of multiple loci; 2) mutations in more than one gene can lead, independently, to the phenotype; 3 ) there are phenocopies of the disorder that are not due to the gene being tested; or 4) the clinical presentation associated with mutation in the gene is poorly defined.

URD 5.2 Analytical Validation

URD 5.2.1 Ultra-rare genetic disease testing requires mutation analysis of specific genes for specific genetic disorders. These tests do not target a particular mutation (apart from custom mutation analysis described in Section URD 10); rather, they are aimed toward detecting the presence of any mutation within the gene being analyzed. For the majority of ultra-rare genetic diseases, the mutations identified are rare and do not occur commonly among patients. A large number of patients have their own private mutations that are not described in any other patients. For this reason, test validation cannot be performed for each and every single mutation that could possibly occur in a particular gene.

URD 5.2.2 As discussed in Section URD 3, several different mutation detection methods exist and are used in different laboratories. The most commonly used method currently is DNA sequencing. Other methods used are described in Section URD 3.3.1 and detailed in the ACMG Standards and Guidelines for Clinical Genetics Laboratories. Regardless of the mutation detection methodology used, all laboratories are required to validate their particular assay first on a number of DNA samples with known sequence information and then on a series of blinded samples containing a diverse array of mutations and sequence changes, as well as blinded specimens with no changes. Blinded samples for validation testing can be obtained from research laboratories as well as from other clinical laboratories that may be offering the test. Records of test validation should be available for review by inspectors from agencies providing laboratory certification.

URD 5.2.3 Given the fundamental rarity of the diseases in question, obtaining multiple blinded samples may not be possible. In this situation, if the test methodology is one in which the laboratory is highly experienced and if successful results have been demonstrated using negative controls, the laboratory may offer a new test while continuing to review and tabulate test results for concordance with clinical diagnosis and family history.

URD 5.2.4 It is equally important in analytical validation to review and document all available knowledge about the locus to be tested, and to update this survey periodically. Critical points to be considered in test design include the existence of any pseudogenes or other homologous genes, and the locations of all reported polymorphisms.

URD 5.2.5 For custom mutation analysis, in which a sequencing-based test is set up to confirm a mutation identified in a research setting, the test can be considered to be "self-validating." When analyzing a sample known to contain the mutation being tested for, this test acts as its own positive control and allows for the analytical validation of the test for that particular mutation. A positive control therefore always exists for the custom mutation analysis tests except in those instances in which a research finding cannot be confirmed. Custom mutation analysis should only be performed by laboratories highly experienced in performing DNA sequence-based tests.

\section{URD 6 QC STANDARDS AND QA PROGRAMS}

URD 6.1 The quality control and quality assurance guidelines published by ACMG for routine molecular genetic tests also apply to ultra-rare disorders, with certain considerations related to the rarity of the disorders. 
URD 6.2 Positive and Negative Controls for Ultra-Rare Disorders

URD 6.2.1. Positive and negative controls for sequencing: Positive controls are not required for sequence-based tests. Negative controls may need to be sequenced if a concurrent comparison is needed to help distinguish between assay artifacts and a true difference.

URD 6.2.2. Positive and negative controls for mutation scanning technologies: A negative control for every fragment should always be tested for comparison to the test sample. It is not necessary or even possible to obtain a positive control for every potential mutation to be detected, but representative positive controls may be run periodically as needed for quality assurance. For some mutation scanning methods, a positive control can be considered to be a sample in which any sequence change (either a mutation or a polymorphism) is known to be present in the amplicon to be tested. As it is unlikely the laboratory will obtain a positive control for every single amplicon being tested for a particular gene, such testing can be performed in the absence of positive controls, but effort should be made to include such controls as they become available.

URD 6.2.3. Positive and negative controls for other methods: Rare or ultra-rare disorders sometimes have hotspots or founder mutations that are amenable to simple tests such as restriction digestion, amplicon length analysis, or allele-specific priming. In such cases, both positive and negative controls are readily available and should always be concurrently tested.

URD 6.2.4 Positive and negative controls for custom (known mutation) tests: In the special situation of custom PCR-based testing for a known mutation, if the mutation was identified elsewhere, the laboratory should make every effort to obtain a positive control and must attach a caveat to the result if one was not available. This control is necessary to confirm the accuracy of the reported mutation, to test the sensitivity of the detection method to be used, and to confirm that the primers can amplify the mutant allele in that family.

URD 6.2.4.1 When known-mutation testing is used for prenatal diagnosis and the fetus is at risk for homozygous recessive mutations, it is equally important to confirm that the primers being used can amplify the parents' normal alleles. Thus both parents must be available as controls, even though another laboratory has already demonstrated that they are heterozygous.

URD 6.3 QA Programs Related to Methodology Shared Among Different Tests

URD 6.3.1 Many different ultra-rare disorder tests performed in a given laboratory may use the same methodology, e.g., sequence analysis. Certain ongoing QA evaluations such as staff competence and software performance can be focused broadly on the methodology level. For example, technicians who are evaluated on a subset of ultra-rare disorder tests may be allowed to perform technical aspects of other tests using the same methodology.

\section{URD 6.4 Proficiency Testing (PT)}

URD 6.4.1 Proficiency testing for ultra-rare disorders is unique in that locus-specific PT is impractical due to: 1) the very small numbers of analyses of any given gene that may be done by a particular laboratory each year; 2) the large number of different genes that may be offered by one laboratory; and 3) the fact that PT for the identification of specific mutations in any given gene is not broadly representative due to the private nature of most mutations in genes associated with ultra-rare disorders. Therefore, PT in the ultra-rare disorders laboratory should be designed to test the proficiency of the laboratory's use of the methods for mutation detection, as well as its ability to detect the different classes of mutations for which services are offered. For example, if the laboratory uses sequencing, restriction digestion, heteroduplex analysis, and quantitative PCR to identify and/or confirm mutations, the PT program for the laboratory should require that 1-3 blinded samples for each methodology should be tested semiannually.

URD 6.4.2 In addition to methodological PT, laboratories are encouraged to establish a locus-specific PT program for any gene that is tested regularly at significant volume. It is appropriate to set up a rotation of locus-specific $\mathrm{PT}$ in proportion to the volume of particular tests performed by the laboratory. A locus-specific PT program can also serve as the methodological PT program.

URD 6.4.3 Specimens for methodological or locus-specific PT programs can be independently arranged on an external or internal basis. Formal PT programs are not available for ultrarare disorders, and partners for interlaboratory exchanges may not be available. If any other laboratories, even internationally, offer testing for the ultra-rare disorder loci, blinded exchanges should be established if possible. If another clinical laboratory partner is not available, specimens could be sent to a genotyping/sequencing core facility in one's institution or elsewhere. Such external comparisons are superior because they not only monitor the reproducibility of results within a laboratory but also permit comparison of alternate test design, including alternate primer binding sites. If the only source of PT specimens is internal, chosen specimens can be resubmitted for a fullblinded analysis. Internally obtained PT specimens can be tested by different technicians and analysts, using any different sequencing apparatus and software that are in place, to evaluate as many components of the testing process as possible.

\section{URD 6.5 Additional QA Opportunities}

URD 6.5.1 Other opportunities to monitor and improve quality may include programs such as tracking the performance of any confirmatory or duplicate tests routinely performed, or 
periodically assessing the observed versus expected ratio of positive results in light of the patient population being tested.

\section{URD 7 TEST INTERPRETATION}

URD 7.1 Interpretation of Sequence Variants (Point Mutations, Small Insertions and Deletions)

URD 7.1.1 Interpretation of sequence variants can be relatively straightforward, but it can also be challenging. The ACMG has developed a position statement entitled "Recommendations for Standards for Interpretation of Sequence Variations" to help laboratories with interpretation and reporting. ${ }^{2}$ Three factors are critical in assessing whether a variant is diseasecausing or benign: 1 ) whether it has been reported in the literature/database; 2 ) whether it has been associated with disease; and 3) whether the type of mutation is expected to cause disease. Thus, the following categories must be defined: 1) reported and known to cause disease; 2) reported and known to be benign; 3) unreported, but expected to cause disease; 4) unreported, and unknown whether it would cause disease; and 5) unreported, but probably does not cause disease.

URD 7.1.2 Once a variant has been identified using a sequencing alignment program, the next step is to identify it with the appropriate nomenclature. ${ }^{3}$ It is critical that laboratories identify and utilize the appropriate cDNA reference sequence for naming mutations, and include this information in the mutation analysis report. If alternative numbering systems or alternate stop codons have been published, the laboratory obviously must bear this in mind when reviewing publications and databases, and also provide a note of explanation in the final report.

URD 7.1.3 Following appropriate naming of the variant, a mutation database search should be done in general databases, including human genome mutation database, ${ }^{4}$ and human genome variation (HGV) databases, ${ }^{5}$ and locus-specific databases. These databases are extremely useful in providing important information to laboratories to allow appropriate interpretation of variants. This information includes a thorough characterization of the variant, including the nucleotide change, the nucleotide position, amino acid changes and codon position, and frequency in the affected population. Other useful information provided in some instances is whether or not the variant has been reported in the unaffected population. Laboratories must explore the databases and literature for assessing variants and determining whether they have been previously reported and thus are recurrent.

URD 7.1.3.1 Public databases of gene-specific mutations may be out-of-date, and thus not contain more recently described mutations. In addition, the databases may reference multiple publications that are actually in conflict, e.g., some claiming that a particular sequence variant is associated with disease, while others claim that the variant is a nondisease associated polymorphism. Databases that are not updated regularly may contain information that is actually incorrect. For example, a particular sequence variant may first be considered benign and later be discovered to be a risk factor. The subsequent papers showing that the variant was actually disease-associated may never have been referenced in the database (or vice versa). The laboratory should exercise caution in relying on databases to interpret the meaning of an observed variant and reflect this in the reporting of results.

URD 7.1.3.2 In numerous cases, laboratories will uncover novel variants during clinical testing. It is important that these variants be as well-defined as possible by the laboratory and that the laboratory report this information back to the databases. Databases are publicly available as a resource. Laboratories are encouraged to develop policies and procedures to routinely send data to the appropriate database.

URD 7.1.4 Variants May Be Classified and Followed Up As Follows:

URD 7.1.4.1. Truncating mutations: Leading to protein truncation-including small insertions and deletions (and nonsense mutations) that create a frame shift-are generally the type that would be interpreted as disease-causing mutations. No additional follow up is necessary.

URD 7.1.4.2. Nonsense mutations: Generally lead to protein truncation, but they can also form cryptic splice sites and may be more difficult to interpret at the molecular level. ${ }^{6}$ Cryptic splice sites can be evaluated by splice site predictor programs as described in Section URD 7.1.4.3.

URD 7.1.4.3. Splicing mutations: Generally occur at the canonical GT:AG splice acceptor/splice donor sites, but they can also occur farther into the intron or within the exon. The use of splice site predictor programs may be useful in predicting whether or not a base change surrounding the splice site will affect splicing. These programs result in a score indicating whether the base change will likely affect splicing. Some examples of splice site predictor programs accessible online, as of September 26, 2005, include the Laboratory of Human Molecular Genetics and Genomic Disorders' Automated Splice Site Analyses, and the Berkeley Drosophila Genome Project. ${ }^{7-9}$ Predictions can vary depending on the algorithm used. As a diagnostic tool, the use of websites and software for this purpose is equivalent to using general reference textbooks. The only way to confirm splicing efficiency predictions clinically is to examine the cDNA by sequencing. This means that an RNA preparation will be necessary, and may require a second specimen from the patient for analysis, except in cases in which laboratories prepare RNA routinely. However, it may be the case that the gene is not expressed in an accessible tissue. 
URD 7.1.4.4. Silent substitutions (third base wobble): Require cautious interpretation because some have been demonstrated to be pathogenic by causing abnormal splicing. ${ }^{6}$

URD 7.1.4.5 Missense substitutions: May actually be normal variants, or may have deleterious effects that can be difficult to recognize, such as causing a loss or gain of protein function due to changes in the secondary structure of a protein or causing spurious splicing of the mRNA due to the formation of a cryptic splice-site. Missense changes that cannot conclusively be determined to be either deleterious or benign should be reported as "sequence change of unknown clinical significance." Some guidelines for interpretation of missense mutations are noted below.

URD 7.1.4.5.1 The most fundamental classification of missense mutations is generally based on whether the amino acid change is conservative or nonconservative. As a rule, nonconservative changes are more likely to be disease-causing mutations. In addition, the laboratory should determine whether or not the amino acid in question is evolutionarily conserved. Evolutionarily conserved amino acids suggest that the amino acid has an important functional role and that any change would be detrimental. The position of the amino acid change within the protein can also be checked. Amino acid changes that occur at known functional domains are more likely to be pathogenic.

URD 7.1.4.5.2 Another useful strategy to assess whether a variant tracks with disease is to analyze other family members, including both those affected and those unaffected. Frequently, follow-up is lost due to various reasons, including family relationships. However, it is recommended that laboratories consider requesting samples from other family members when appropriate.

URD 7.1.4.5.3 While these approaches are classical, there are newer ways to address this question, including using predictive software tools for protein modeling to determine how changes might be detrimental to protein function. Another approach is use of predictive software tools, such as evolutionary trace (Lichtarge Laboratory, Baylor College of Medicine), to determine how likely the change is to affect protein function. Some of these tools are presently in evaluation and may not be available for clinical laboratories at this time.

URD 7.1.4.5.4 The ultimate test for any of these unclassified small variants would be the development of a functional assay to test whether or not the change affects protein function. There are very few examples of such a test in a clinical laboratory at the time of this writing. Functional studies are usually beyond the scope of the clinical laboratory, but "partnering" with research laboratories for this purpose may be feasible.

URD 7.1.4.6 Single nucleotide polymorphisms (SNPs) are recurring variations in coding or noncoding regions that have no known or obvious disease-causing effect. If a single nucleotide substitution is discovered in a patient, the laboratory must determine if it is a recognized SNP of no clinical significance; this can be done by searching public SNP databases ${ }^{10,11}$, by consulting researchers studying that gene, and/or by examining a significant number of normal sequences prospectively or retrospectively. If the change is concluded to be a common benign variant, the basis of this conclusion should be stated in the report. If the minor variant is seen in the general population but is reported to be over-represented in the affected population, a citation of the study should appear in the report. If the change cannot be categorized as a known benign SNP, it should be reported as an "unclassified variant" or "variant of unknown significance.”

URD 7.1.4.6.1 The presence of nondisease associated SNPs in a sequence should be reported because the allele may be discovered in the future to be disease-associated. Furthermore, heterozygosity at any position in any gene is often useful and can serve: 1) to assure that both alleles have been amplified and are represented in the analysis; 2) as markers for linkage analysis in families where applicable; 3 ) to evaluate a prenatal sample for the presence of maternal cell contamination; and 4) to address issues of sample-switching or nonpaternity/maternity. For these reasons, it is recommended that the presence of heterozygosity at SNP positions be noted in the mutation analysis report. Either the location of the SNP (e.g., ivs2-106), or the location along with the actual genotype (e.g., ivs2-106, g/a) can be noted. One suggested format for commenting on SNPs without distracting from the overall "positive" or "negative" results would be to address SNPs in a separate section of the report or footnote.

URD 7.1.4.6.2 The observation of apparent homozygosity for a rare SNP allele with no obvious pathogenic consequences may be worthy of comment, since such variants could later be determined to have some associated risk, but it would be inappropriate to raise extreme concern. More to the point, in the special situation when the possibility of a large deletion is already being considered in a nonconsanguineous family due to the absence of heterozygous positions, the detection of apparent homozygosity for a rare SNP allele could be another indication of a deletion and possibly serve as a marker for parental studies.

\section{URD 7.2 Interpretation of Gross Alterations}

URD 7.2.1 Gross alterations (deletions, duplications, or rear-

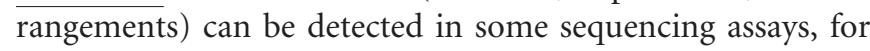
example by failure to amplify an X-linked gene in a male or by detection of homozygosity for a mutation or polymorphism in a proband that is not confirmed in the patient's parents. Numerous other strategies mentioned in section URD 3.4.2 are designed for this purpose. With such tests, any result that is different from that obtained for the normal control is indicative of a gross alteration and can usually be interpreted as pathogenic. Results that do not differ from the normal control are interpreted as 
grossly normal. In the case of a normal result, it is important to state the sensitivity of the assay within the final report.

URD 7.2.2 Gross alterations are generally under-represented in mutation databases. This is partly due to such mutation types not being routinely tested for and reported.

URD 7.2.3 When a gross alteration is identified, its exact boundaries are not generally defined. As a result, it is difficult to assign a precise nomenclature. Such abnormalities are generally described simply as, for example, "deletion of exon (\#) in gene (name)."

\section{URD 7.3 Interpretation of a Normal Result}

URD 7.3.1 When a normal result is obtained, it is important to refer to the analytical sensitivity of the test and to state that an undetected mutation may be present. This could be due to: 1 ) the nature of the mutation, e.g., deletions; 2 ) the presence of mutations outside of the coding regions that were not tested, e.g., in the promoter region; 3 ) the presence of a mutation in a totally different gene; 4 ) the presence of unexpected variation in a particular patient at a primer binding site, leading to alleledrop-out or preferential amplification of a competing allele; or 5) mosaicism.

\section{URD 8 TYPE OF TESTING}

URD 8.1 The type of testing depends upon the type of disorder and inheritance pattern. Rare disorder testing may include diagnostic testing, carrier testing, and prenatal testing. Population screening, such as carrier testing for CF, is less likely for ultrarare disorders, although screening for a mutation in the spouse of an individual known to be a heterozygote for a rare autosomal recessive disorder is occasionally requested. Initial testing may include confirmation of research findings in a clinical laboratory.

URD 8.2 Prenatal testing carries high liability and should be undertaken with caution, particularly for ultra-rare disorders for which limited clinical validation data are available. Specific guidelines for prenatal testing should be followed (see Section G19 of the ACMG Standards and Guidelines for Clinical Genetics Laboratories).

\section{URD 9 PRE- AND POST-TEST ISSUES}

URD 9.1 Pretest and post-test issues such as specimen requirements, transport, patient identification, family identification, criteria for rejection, etc., are generally the same for ultra-rare disorders as for other molecular genetic tests, with the following points of emphasis:

\section{URD 9.2 Pre-Test Considerations}

URD 9.2.1 Test description and/or informed consent document: A test description and/or test-specific informed consent document can help both the patient and the ordering physician understand the appropriate uses and limitations of the test. The document should define the name of the gene or genes to be tested; the regions (exons) to be tested; the clinical disorders associated with that gene; and the usual indications for testing; such as differential diagnosis, carrier test, or prenatal diagnosis. The document should state both the clinical sensitivity and analytical sensitivity, for example " $60 \%$ of patients with this disease have mutations in the XYZ gene. Over $90 \%$ of published mutations in the XYZ gene, to date, would be detected by sequence analysis of exons 2,3 , and $4 . "$

URD 9.2.2 Who can submit tests: Like all genetic tests, URD tests may be ordered by a physician of any specialty and may be submitted as referred tests from general pathology laboratories. Patients cannot submit tests directly.

URD 9.2.3 Clinical history and family history: Laboratories should request any background information that is necessary for interpreting the test result. At a minimum, this information would typically indicate if the patient is affected with the disorder or has a positive family history, and whether the family has a known mutation. In some disorders, priorities for the analysis of different genes or exons may depend on the phenotype, the ethnic group, or the inheritance pattern.

URD 9.2.4 Special preanalytical criteria for identification of specific mutations identified elsewhere:

URD 9.2.4.1 Information provided about the mutation must be sufficiently unambiguous to identify the gene and nucleotide(s) to be tested. Missense and stop mutations should be described at the DNA level as well as the protein level. Reference sequences should be given. Numbering conventions, such as with or without a leader peptide, should be stated. Surrounding sequence is very helpful.

URD 9.2.4.2 The importance of retesting a positive specimen should be presented to the referring physician. This practice not only guards against communication errors, historical changes in the numbering of exons, and inconsistency in the numbering of nucleotides and codons, but also allows the laboratory to absolutely rule out allele dropout of the abnormal allele which can occur through family-specific polymorphisms in primer sites, thus providing a level of customized QC not otherwise available. Although allele dropout is rare, the ease of doing this simple QC step makes it compelling.

URD 9.3 Post-Test Considerations

URD 9.3.1 Interpretation of the clinical significance: See section URD 7.

URD 9.3.2 Report contents: Elements of routine molecular laboratory reports as presented in the ACMG Standards and Guidelines for Clinical Genetics Laboratories, section G17, are applicable to ultra-rare disorders as well. They include patient 
name; other patient identifiers; specimen type; draw date; received date; accession number; family number (if used); test ordered (disease name and gene name); clinical status or reason for test; method; result; interpretation; limitations; recommendations. Tests for ultra-rare disorders or any sequencing test should identify the reference sequence used and the location in that sequence of nucleotide 1 and codon 1 in relation to the initiator ATG. Any mutations or polymorphisms identified should be stated using standard nomenclature on both the DNA level and the protein level.

URD 9.3.3 Transmission of report Laboratories should be aware that it is increasingly common for hospital and reference lab databases to truncate or summarize lengthy results. It is wise to provide a succinct, easily excerpted statement of critical information, such as "negative with limitations," indicating that important comments are included.

URD 9.3.4 Sample Reports: (See Appendix)

\section{URD 10 CUSTOM ANALYSIS}

URD 10.1 Custom mutation analysis applies to situations in which a disease mutation has been identified in a patient in a research setting and needs to be confirmed in the clinical setting so that the results may be: 1) released to the patient/family and/or referring physician for purposes of diagnosis and treatment; 2) used for further testing in other family members to determine mutation status; and 3) utilized in prenatal testing situations. Custom mutation analysis applies to those genetic disorders in which only rare private mutations have been described (the majority of ultra-rare genetic disorders), for which testing is not routinely available, and to those more common genetic disorders (e.g., cystic fibrosis, Duchenne muscular dystrophy, Canavan disease, to name a few) in which an uncomamon/rare mutation, not routinely tested for, is found to be present in a patient or family. GeneTests ${ }^{12}$ lists laboratories that provide a custom mutation analysis service, i.e., laboratories that will clinically confirm mutations identified in research laboratories.

URD 10.2 The clinical validation of custom tests for ultra-rare disorders or other private mutations is the obligation of the researcher who performed the initial study. In performing a confirmation of a research laboratory finding, the clinical laboratory is responsible for only the analytical validation, as described in Sections URD 5.2.5 and URD 10.3. The laboratory report may address only the presence or absence of the mutation and refer to the researcher's interpretation for causative association with disease.

URD 10.3 For purposes of mutation confirmation, it is essential that the test be performed on a new sample obtained from the patient and sent directly to the clinical laboratory, i.e., not a sample that was received and processed by the research laboratory that originally identified the mutation. For analytical validation, it is important for the clinical laboratory to confirm the mutation in the original proband prior to performing any testing on additional family members, and particularly prior to any prenatal testing. In the event of limited time it is acceptable to perform the test on the proband (for confirmation purposes) and the additional family member/prenatal sample at the same time. If the mutation finding cannot be confirmed by the clinical laboratory on the original proband, this needs to be stated in the patient's report and no reporting can be made of any additional family members/prenatal samples until the discrepancy in the proband's result has been clarified. In some instances the original proband is deceased and mutation confirmation cannot be performed on a new sample. In such instances the following options can be pursued:

URD 10.3.1 Family members who are obligate carriers of the mutation can be used to establish the clinical mutation confirmation in that family.

URD 10.3.2 If no family member can be identified in whom mutation confirmation can be performed, then mutation testing on at-risk family members is performed in the absence of formal mutation confirmation. If the mutation is found to be present in any family member being tested, the familial mutation for that family is confirmed and can be reported. However, if the family member being tested is found not to carry the mutation, it is very important to state in the report that a positive control from that family was not available for the mutation being tested. The caveat should emphasize that the results being reported are dependent on the accuracy of the information received from the previous laboratory.

URD 10.3.3 At the very minimum, in the absence of a new positive control specimen from the family, it is important to obtain a DNA aliquot from the previous laboratory to serve as a positive control for a custom test. This permits technical validation of the primers and conditions for detection of that mutation, although it does not rule out possible misidentification of that original research specimen.

URD 10.4 It is important that the clinical laboratory has sufficient information about the mutation to be tested prior to setting up the mutation-specific assay. Certain mutations may not be amenable to detection using the techniques available to the clinical laboratory, for example, the identification of large intragenic gene deletions by routine PCR-based methods. Information regarding the mutation to be tested, what gene it occurs in, where it occurs in the gene, and, if possible, assay conditions used by the research laboratory to identify the mutation should be provided to the clinical laboratory prior to developing the mutation-specific assay. It is important to make sure that the mutation nomenclature provided to the clinical laboratory is clear and unambiguous. 
URD 10.5 For single base changes and small deletions/insertions, DNA sequencing is the appropriate technique to be used for confirming a mutation in a custom mutation analysis. A PCR amplicon is generated that contains the region of the mutation and is sequenced in a double-stranded fashion. It is important to keep in mind that certain areas of the genome are harder to analyze (such as GC-rich regions or regions of repetitive DNA) and therefore sufficient time needs to be allocated to setting up custom mutation tests. Four to six weeks is generally adequate time to set up such tests and takes into account potential problems that may be encountered. Once a mutation-specific assay has been developed, testing of additional family members is easier and faster (2-3 weeks) and may be accomplished by a less direct method, such as restriction digestion or a scanning technology.

URD 10.6 Confirmation of Negative Results from a Research Laboratory

URD 10.6.1 Mutation confirmation for custom genetic tests usually is requested to confirm a positive result identified in a research laboratory. However, to ensure the accuracy of both positive and negative results from a research laboratory, the research laboratory should be encouraged to have the clinical laboratory confirm the result on all family members, regardless of whether the family member was found to have the mutation or not. In such cases, analysis in the clinical laboratory will likely only be performed for the specific mutation in the family that was identified by the research laboratory, and the reported results should reflect that a full analysis of the gene was not accomplished. Thus, mutations elsewhere in the gene would not have been identified by the analysis performed by the clinical laboratory.

URD 10.6.2 It is rarely requested that a clinical laboratory confirm negative results for a research participant on whom no mutation was found after a thorough research evaluation of the disease gene. In most cases, confirmation of negative research results in this situation will need to wait until a clinical laboratory offers testing of the disease gene on their usual test menu. Research laboratories should be encouraged to translate research testing into a clinical laboratory as soon as the test is deemed clinically valid. Once clinical testing is available, researchers should be encouraged to pursue clinical confirmation of negative results.

\section{URD 11 PITFALLS}

URD 11.1 The use of different mutation nomenclatures by different laboratories when numbering is performed using different reference sequences: This is extremely important for custom mutation testing purposes to enable the correct region of the gene to be targeted for mutation testing. It is important to make sure that there is no discrepancy in the mutation nomenclature information and for this reason, adhering to the recommendations made for mutation nomenclature is strongly recommended..$^{3,13}$

URD 11.2 Observation of a rare mutation in the homozygous state in an autosomal recessive disease situation: In the absence of known consanguinity, the possibility of a deletion on one allele resulting in hemizygosity for the particular mutation (as opposed to homozygosity) should be considered. Similarly, interpretations of homozygosity for any rare polymorphism at any diploid locus should also be considered as possible hallmarks of deletions.

URD 11.3 Observation of homozygosity, or inability to confirm a mutation identified in a research laboratory: This result may be due to the presence of a polymorphic position within the primer sequence being used to amplify the DNA fragment. In such cases, it is necessary to repeat the analysis using a second set of primers positioned away from the original primer pair. It is useful, but far from foolproof, to check SNP databases when designing primers. Laboratories should use disclaimers on their reports that identify such pitfalls.

URD 11.4 Interpretation of mutation/sequence change findings can be difficult (e.g., missense changes, noncoding region changes, silent changes). The presence of a database of mutations and polymorphisms for all genes being tested will help. In some cases it is necessary to defer to the research laboratory from which the mutation information originated when interpreting such results. Clinical laboratories should be cautious about deferring to research laboratories.

\section{ACKNOWLEDGMENTS}

The authors wish to acknowledge the participation of Drs. David H. Ledbetter, PhD (Emory University School of Medicine) and D. Joe Boone, $\mathrm{PhD}$ (Centers for Disease Control) in the development of the content of these guidelines.

\section{References}

1. National Human Genome Research Institute. http://www.genome.gov/10001733 Accessed September 26, 2005.

2. ACMG Laboratory Practice Committee Working Group. Recommendations for standards for interpretation of sequence variations. Genet Med 2000;2:302-303.

3. Antonarakis SE. Recommendations for a nomenclature system for human gene mutations: Nomenclature Working Group. Hum Mutat 1998;11:1-3. www. genomic.unimelb.edu.au/mdi/rec.html. Accessed September 26, 2005.

4. Institute of Medical Genetics. Human Genome Mutation Database http://www. hgmd.cf.ac.uk/hgmd0.html. Accessed September 26, 2005.

5. Human Genome Variation Society. Variation databases http://www.genomic unimelb.edu.au/mdi/dblist/dblist.html. Accessed September 26, 2005.

6. Cartegni L, Chew SL, Krainer AR. Listening to silence and understanding nonsense: exonic mutations that affect splicing. Nat Rev Genet 2002;3:285-298.

7. Cartegni L, Wang J, Zhu Z, Zhang MQ, Krainer AR. ESEfinder: a web resource to identify exonic splicing enhancers, release 2.0. Nucleic Acid Research 2003; 31:35683571. http://rulai.cshl.edu/tools/ESE/. Accessed September 26, 2005.

8. Laboratory of Human Molecular Genetics and Genomic Disorders. Automated Splice Site Analyses https://splice.cmh.edu. Accessed September 26, 2005.

9. Splice Site Prediction by Neural Network. Berkeley Drosophila Genome Project http:// www.fruitfly.org/seq_tools/splice.html. Accessed September 26, 2005.

10. The SNP Consortium LTD Single Nucleotide Polymorphisms for Biomedical Research http://snp.cshl.org/. Accessed September 26, 2005 
11. National Library of Medicine www.ncbi.nlm.nih.gov/entrez/query.fcgi?db $=s n p$. Accessed September 26, 2005.

12. National Institutes of Health.GeneTests. http://www.genetests.org. Accessed September 26, 2005

13. den Dunnen JT, Antonarakis SE. Mutation nomenclature extensions and suggestions to describe complex mutations: a discussion. Hum Mutat 2000;15:7-12.

\section{APPENDIX}

Sample Laboratory Reports For Ultra-Rare Disease Testing

\section{SAMPLE 1: Complete Gene Sequencing with Negative Results and Heterozygous Positions}

Test: Sequence analysis of the XXX gene

Indication for Testing: Suspected diagnosis of syndrome

Method: The 20 coding exons and the flanking intronic regions of the gene were amplified by PCR and sequenced in two directions. The reference mRNA sequence is NM_xxxxxxxx, with codon 1 corresponding to the start ATG and nucleotide 1 corresponding to the A.

Result: No XXX gene mutation was detected.

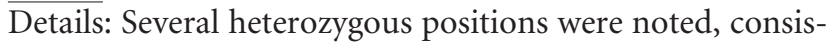
tent with the detection of two normal alleles. At codon 255, both TAT and TAC were present. This is a known polymorphism of no apparent clinical significance, since both sequences encode tyrosine. At codon 871, both CCA (proline, P) and CGA (arginine, R) were present. P871R is reported to be a nondisease associated normal variant (Author et al., 1999). Heterozygosity was also seen at a noncoding position, c.428-15 (IVS3-15), which has no apparent effect. The presence of these heterozygous positions confirms that two alleles were present and excludes the possibility of a complete deletion of one allele.

Interpretation: No mutation associated with syndrome was detected in the gene of this patient. This result does not exclude the diagnosis of this syndrome. Mutations in are found in $60 \%$ of patients with syndrome and a positive family history, and in 35\% of isolated cases (Author et al., 2000). The interpretation is based on the current understanding of the genetics of syndrome.

Limitations: Only the coding regions of the XXX gene and immediate flanking intron sequences were examined. Changes in the promoter region, farther into the introns, or in other noncoding regions of the gene, would not be detected. The sensitivity of DNA sequencing is over $99 \%$ for the detection of nucleotide base changes, small deletions, and insertions in the regions analyzed. Mutations in genes other than XXX would not be identified. Multiple exon deletions, multiple exon insertions, and complete deletion of one allele may not be identified using these methods. Other types of rare genetic variation can interfere with this analysis.

References:

\section{SAMPLE 2: Complete Gene Sequencing with Negative Results and No Heterozygous Positions}

Test: Sequence analysis of the XXX gene
Indication for Testing: Suspected diagnosis of syndrome

Method: The 12 coding exons and the flanking intronic regions of the XXX gene were amplified by PCR and sequenced in two directions. The reference mRNA sequence is NM_xxxxxxxx, with codon 1 corresponding to the start ATG and nucleotide 1 corresponding to the $\mathrm{A}$.

Result: No XXX gene mutation was detected.

Details: No heterozygous positions were observed. If present, heterozygous normal polymorphic positions serve to rule out gross gene deletions. Deletions are known to occur in syndrome in over $10 \%$ of cases. Furthermore, this patient's sequence included an apparently homozygous $G$ at position c.428-34 (IVS3-34). This is a change from the prototypic $\mathrm{T}$ that we have not observed in over 100 control and patient sequences. The presence of G at IVS3-34 is not thought to be a causative mutation, but it can be taken as an additional indication for deletion testing, since a rare sequence change is unlikely to appear homozygous unless the patient's parents are consanguineous.

Interpretation: No mutation associated with syndrome was detected in the XXX gene of this patient. This result does not exclude the diagnosis of this syndrome. Sequence mutations in XXX are found in $60 \%$ of patients with syndrome and a positive family history, and in 35\% of isolated cases (Author et al., 2000). Testing of the patient's parents for the IVS3-34 can be performed to clarify the presence of a deletion in this patient. Alternatively, deletion analysis for this gene can also be considered if available. This interpretation is based on the current understanding of the genetics of syndrome.

Limitations: Only the coding regions of the XXX gene and immediate flanking intron sequences were examined. Changes in the promoter region, farther into the introns, or in other noncoding regions of the gene, would not be detected. The sensitivity of DNA sequencing is $99 \%$ for the detection of nucleotide base changes, small deletions and insertions in the regions analyzed. Mutations in genes other than XXX would not be identified. Multiple exon deletions, multiple exon insertions, and complete deletion of one allele may not be identified using these methods. Other types of rare genetic variation can interfere with this analysis.

References:

\section{SAMPLE 3: Gene Scanning and Sequencing with Positive Results}

Test: Mutation analysis of the XXX gene

Indication for Testing: Suspected diagnosis of syndrome

Method: The 20 coding exons and the flanking intronic regions of the XXX gene were amplified by PCR and screened for mutations using denaturing high performance liquid chromatography (dHPLC). Amplicons showing variations were sequenced in two directions. The reference mRNA sequence is 
NM_xxxxxxxx, with codon 1 corresponding to the start ATG and nucleotide 1 to the $\mathrm{A}$.

Result: Positive for two mutations: S320P and p.L1061fsX4.

Details: S320P (serine to proline at amino acid 320) is caused in this patient by a $\mathrm{T}$ to $\mathrm{C}$ substitution at nucleotide position c.958. This mutation has not been reported in other syndrome patients to our knowledge, but it causes a nonconservative amino acid substitution in a critical domain, at a position where serine is invariant in all vertebrates. S320P has not been reported as a normal variation and has not been observed in our analysis of over 50 normal alleles. Thus, S320P is likely to be a causative mutation for syndrome, although functional studies have not been done to prove this conclusively. p.L1061fsX4 describes a frameshift mutation in the leucine (L) codon 1061 caused by deletion of the normal $\mathrm{T}$ at position c.3183. The frameshift leads to a premature termination 4 codons downstream. p.L1061fsX4 has been previously reported in patients with syndrome (Author et al., 1995).

Interpretation: S320P and p.L1061fsX4 are assumed to be the cause of syndrome in this patient. The detection of two mutations in the XXX gene confirms the diagnosis and provides specific mutation information that can be used for carrier testing and prenatal diagnosis in this family. In this autosomal recessive disorder, the parents of this patient are each assumed to carry one mutation, although new mutations are possible. This interpretation is based on our current understanding of the genetics of syndrome.

Limitations: The two mutations found are assumed to be on opposite chromosomes, but this cannot be proven by sequencing. This assumption must be confirmed by testing the parents of this patient prior to any additional testing in this family.

References:

\section{SAMPLE 4: Complete Gene Sequencing with Results of Unknown Significance}

Test: Sequence analysis of the XXX gene

Indication for Testing: Suspected diagnosis of syndrome

Method: The 5 coding exons and the flanking intronic regions of the XXX gene were amplified by PCR and sequenced in two directions. The reference mRNA sequence is NM_xxxxxxxx, with codon 1 corresponding to the start ATG and nucleotide 1 to the $\mathrm{A}$.

Result: A variation of unknown significance was identified: c.978 C to T (S326S). No other differences from the prototypic normal sequence were observed.

Details: This patient is heterozygous for a $\mathrm{C}$ to $\mathrm{T}$ substitution at nucleotide c.978, resulting in AGT instead of AGC at codon 326. Both sequences encode serine (S). This sequence change does not appear to have been previously described in patients with syndrome and has also not been described as a known polymorphism (benign sequence change) in the XXX gene. Since the amino acid is not changed and a spurious splice site does not appear to be introduced, it is possible that this change is nonpathogenic, but functional studies have not been performed to prove this conclusively.

Interpretation: No obvious pathogenic mutation was discovered but an inconclusive finding is reported. Over $80 \%$ of patients with classical ___ syndrome have obvious pathogenic sequence mutations in the XXX gene. Testing of the patient's parents for the c.978C $>$ T change, along with a thorough physical examination for mild signs of syndrome, may help determine the pathogenic nature of this finding.

Limitations: Only the coding regions of the XXX gene and immediate flanking intron sequences were examined. Changes in the promoter region, farther into the introns, or in other noncoding regions of the gene would not be detected. The sensitivity of DNA sequencing is over $99 \%$ for the detection of nucleotide base changes, small deletions and insertions in the regions analyzed. Mutations in genes other than XXX would not be identified. Multiple exon deletions, multiple exon insertions, and complete deletion of one allele may not be identified using these methods. Other types of rare genetic variation can interfere with this analysis, but complete absence of one copy of the XXX gene is ruled out by the observation of heterozygosity at one or more positions.

References:

\section{SAMPLE 5: Custom Testing for a Known Mutation with Positive Results}

Test: Custom analysis for a G403R mutation in exon 8 of the $\mathrm{XXX}$ gene

Indication for Testing: Family history of syndrome. The mutation G403R was reported to have been detected in this family in the research laboratory of Dr. , who has referred this patient to us for clinical testing.

Method: Research results were provided indicating that a member of this family has a GGC to CGC mutation in codon 403 (glycine to arginine) of the XXX gene, using mRNA reference sequence NM_xxxxxxxx and assigning codon 1 to the start ATG with the inclusion of the cleaved signal peptide. The $\mathrm{G}$ to $\mathrm{C}$ nucleotide change corresponds to position c.1207 in the mRNA. Exon 8 was amplified by PCR and sequenced bi-directionally. Analysis was performed on a separate blood sample from the patient sent directly to our laboratory. (In the event a separate blood sample from the affected proband is not available: A DNA sample was provided by the researcher as a positive control, although an independent new positive control sample was not available).

Result: The G403R mutation was found in the heterozygous state in this patient.

Details: Testing of the patient's blood sample (or: positive control DNA sample provided by the researcher) allowed us to demonstrate that the test method we designed could detect the reported G403R mutation. The detection of this mutation in the patient confirms the research laboratory's finding in this family. 
Interpretation: This patient has the G403R mutation previously reported to be in her family. This mutation has been reported in the literature to be deleterious (cite reference), and thus is interpreted as a disease-causing mutation. Genetic counseling is recommended for this individual.

Limitations: Only the region immediately surrounding codon 403 of the XXX gene was examined. Changes elsewhere in the gene, or in other genes, would not have been detected. (In the event a separate blood sample from the affected proband is not available: As a separate patient sample could not be tested, the presence of a sample mix-up in the research laboratory cannot be ruled out.)

References:

\section{SAMPLE 6: Custom Testing for a Known Mutation with Failure to Confirm}

Test: Custom analysis for an R100X mutation in exon 4 of the XXX gene

Indication for Testing: Family history of syndrome. The mutation R100X was reported to have been detected in this patient in the research laboratory of Dr. , who has referred the family to us for clinical testing. This patient is being tested as a positive control.
Method: Research results were provided indicating that this patient has an AGA to TGA mutation in codon 100 (arginine to stop) of the XXX gene, using mRNA reference sequence NM_xxxxxxxx and assigning codon 1 to the initiator ATG. The A to T nucleotide change corresponds to position c.298 in the mRNA. Exon 4 was amplified by PCR and sequenced bi-directionally.

Result: Unable to confirm the presence of any mutation in co $\overline{\text { don R1}} 00$.

Details: Only the normal exon 4 sequence was seen. No heterozygous positions were detected elsewhere in the exon or flanking intronic sequence; such positions, if present, would have confirmed that two alleles were amplified. The same result was obtained using an alternate set of primers.

Interpretation: This patient is demonstrating discordant results between our laboratory and the research laboratory. Rare genetic variants can interfere with this type of analysis, particularly in primer binding sites, so two different primer sets were used. Neither set detected the reported mutation. Until this discordance is resolved, this test will not be available for additional members of this family.

Limitations: Only exon 4 of the XXX gene was examined. Changes elsewhere in the gene, or in other genes, would not have been detected.

References: 